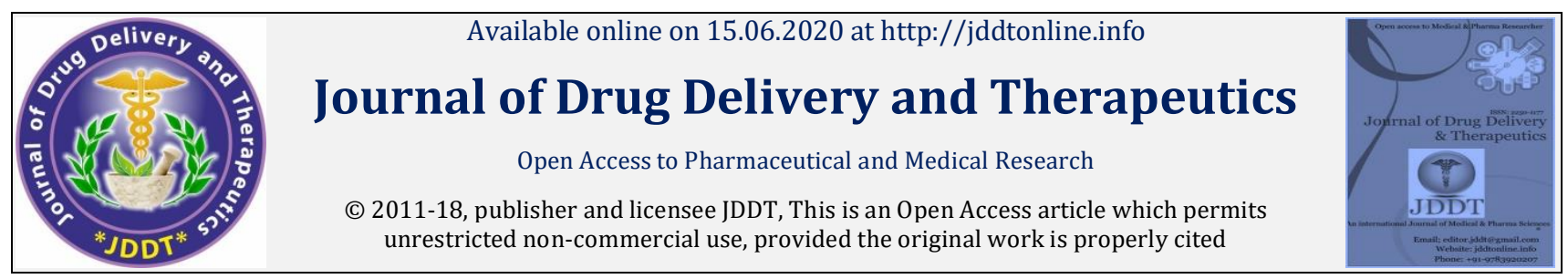

Open 1 Access

Review Article

\title{
Silymarin Loaded Novel Drug Delivery for Oral and Topical Administration
}

\section{Gorhe Ankitaa, Kulkarni Abhijeet ${ }^{b}$, Kandalkar Pallavic, Jagtap Snehald}

a Department of Pharmaceutical Quality Assurance, Sanjivani College of Pharmaceutical Education and Research, Kopargaon- 423601, Maharashtra, India.

b Department of Pharmaceutical Quality Assurance, Sanjivani College of Pharmaceutical Education and Research, Kopargaon- 423601, Maharashtra, India.

c Department of Pharmaceutical Quality Assurance, Sanjivani College of Pharmaceutical Education and Research, Kopargaon- 423601, Maharashtra, India.

d Department of Pharmaceutical Quality Assurance, Sanjivani College of Pharmaceutical Education and Research, Kopargaon- 423601, Maharashtra, India.

\begin{abstract}
Silymarin is polyphenolic flavonoid obtained from the seeds of silybum marianum plant. It has various pharmacological properties such as hepatoprotective, anti-inflammatory, antioxidant, anti-carcinogenic, hypolipidemic properties. Silymarin has recently reported to be neuroprotective agent against neurodegenerative disease such as Alzheimer, Parkinson's and cerebral ischemia. It contains eight active components, among which silibinin is the most active component. However, silymarin is BCS class II drug which having poor bio availability due to extensive phase II metabolism, poor aqueous solubility, low permeability across intestinal epithelial cells and rapid excretion in bile and urine. Therefore, it is necessary to understand all formulations and analytical aspects including all possible future prospects. In this review a potential approach to enhance solubility, bioavailability and to develop a robust formulation is studied. The number of studies describes novel drug delivery system (NDDS) based formulations have been significantly increased. The raise in novel drug delivery exploitation is essentially due to defeated barriers within technological process of lipid based nanoparticles formulations and increased knowledge of underlying mechanisms of transport of NDDS via different route of administration. This review focuses on pharmacological properties of silymarin, challenges, benefits and application of novel drug delivery system. To reduce the adverse effects and toxicities novel drug delivery will be an attractive approach of current therapies.
\end{abstract}

Keywords: Silymarin, route of administration, novel drug delivery, bioavailability, solubility

Article Info: Received18 March 2020; Review Completed 18 May 2020; Accepted 24 May 2020; Available online 15 June 2020

Cite this article as:

Gorhe A, Kulkarni A, Kandalkar P, Jagtap S, Silymarin Loaded Novel Drug Delivery for Oral and Topical Administration, Journal of Drug Delivery and Therapeutics. 2020; 10(3-s):262-270 http://dx.doi.org/10.22270/jddt.v10i3-s.4125

*Address for Correspondence:

Dr. Abhijeet Kulkarni, Department of Quality Assurance Techniques, Sanjivani College of Pharmaceutical Education and Research, Kopargaon- 423601, Maharashtra, India

\section{INTRODUCTION:}

Silymarin is obtained from the extracts of seeds and fruits of Silybum marianum plant it's common name is milk thistle and it is chemically known as 2-(2,3-dihydro-2-(4-hydroxy3-methoxyphenyl)-3-(hydroxymethyl)-1,4-benzodioxin-6yl)-2,3-dihydro-3,5,7-trihydroxy4H-1-benzopyran-4-one1. It is one of the oldest and thoroughly researched plants of ancient times used as herbal medicine and food supplement for the treatment of various diseases associated with liver and gallbladder, hepatitis, cirrhosis, jaundice and protection against amanita phalloides mushroom and other toxin poisonings. The silymarin is composed of three isomer flavonolignans: silybin, silydianin, isosilibinin and silychristin ${ }^{2}$. Silybin (silibinin) is the most active component of silymarin ${ }^{3}$. Silybin is the mixture of two diastereomers A and $\mathrm{B}$ in approximately $1: 1$ propoportion containing therapeutic properties such as antioxidant, antiinflammatory, anti-carcenogenic, neuroprotective, hepatoprotective, cardioprotective properties.

In polar aprotic solvents (eg. acetone, N, Ndimethylformanide, and tetrahydrofuran) silybin is highly soluble, in polar protic solvents (e.g ethanol and methanol) it is poorly soluble and in non-polar solvents (eg. chloroform and petroleum ether) it is insoluble. The pharmacokinetics studies showed that the oral administration of silymarin is only $23-47 \%$ absorbed from gastrointestinal tract where it undergoes enterohepatic circulation ${ }^{4,5}$. It is metabolized by CYP450-2C8 to mono and dihydroxy silybin (minor) and o- 
demethylated silybin (major) metabolites ${ }^{6}$. During the phase II metabolism multiple conjugation reactions are observed it includes formation of silybin monoglucoronide, silybin diglucoronide, silybin monosulfate, and silybin diglucoronide sulfate ${ }^{7}$. The small amount of absorbed silybin is excreted in kidney and about $18 \%$ is excreted in the bile after conjugation with sulfate and glucuronide. The reported clearance half-life of silymarin is 6-8 hours ${ }^{8}$. Poor aqueous solubility, high metabolism, poor penetration across epithelial cells, rapid systemic excretion these are main reasons for limited bioavailability of silybin. To overcome these issues, novel drug delivery has shown great potential using different formulations like liposomes, microspheres, solid dispersion, emulsions, dendrimers, solid lipid nanoparticles, nanosuspension, nanocrystals, inclusion complex, micelles, to improve the aqueous solubility, penetration ability and to enhance bioavailability.

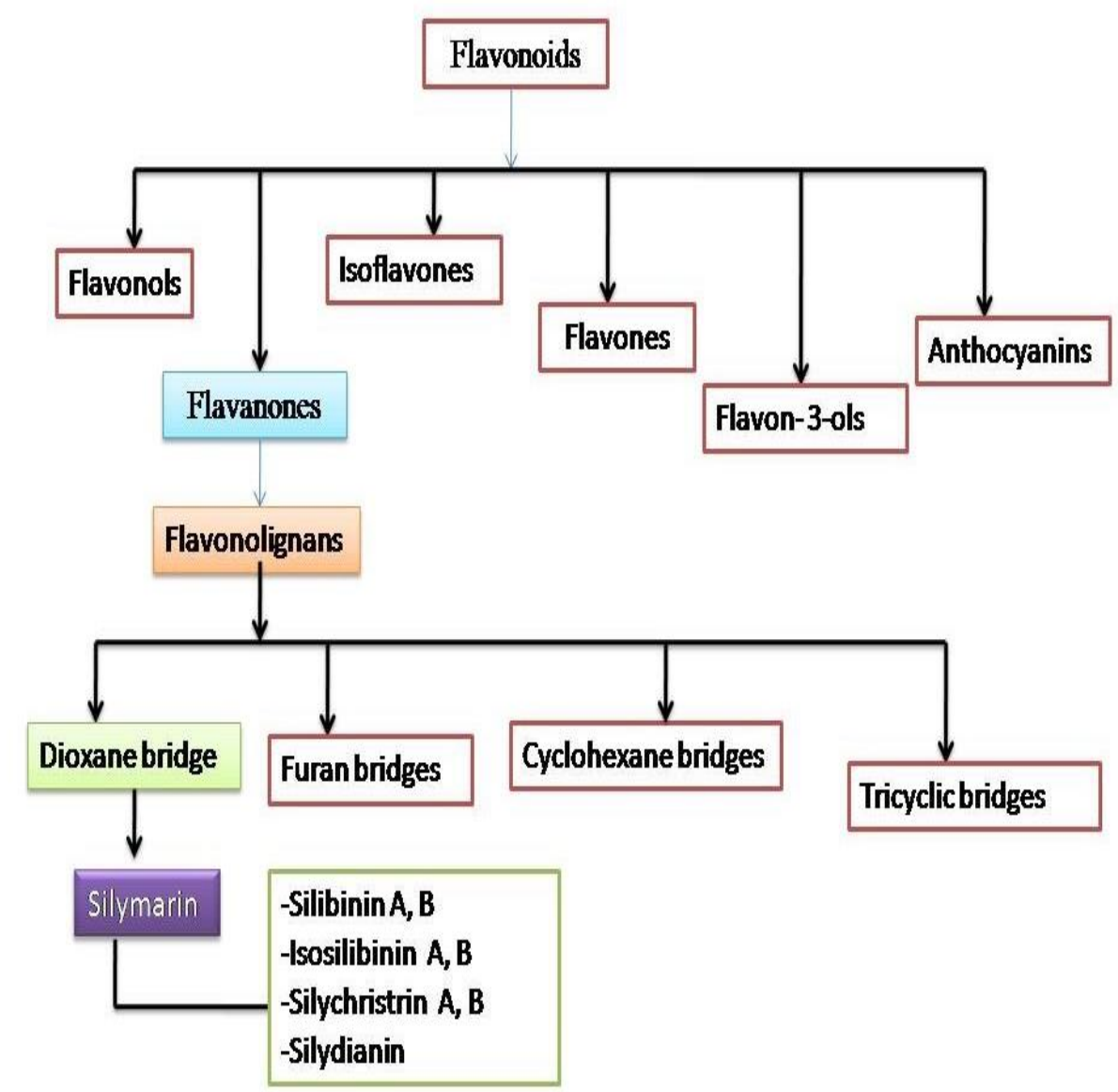

Figure 1: Classification of flavonoids

\section{PHARMACOLOGICAL PROPERTIES:}

Silymarin shows antioxidant properties and acts as a free radical scavenger that induces lipid peroxidation and also influences enzyme systems associated with glutathione and superoxide dismutase ${ }^{9}$. In pre-clinical study silymarin and silybin are agents found to be liver protective in mouse and rats against hepatotoxicity induced by different agents such as carbon tetrachloride, ethanol intoxication, cisplatin, acetaminophen, thioacetamide ${ }^{10}$. The silymarin shows action on lung cancer, breast cancer, ovarian cancer, cervical cancer, skin cancer, prostate cancer, liver carcinoma, bladder cancer. Silybin induced MCF7 breast cancer cells to undergo autophagic cell death and observed formation of autophagy related genes i.e Atg12-Atg5, Beclin-1 upregulation and Bcl-2 downregulation ${ }^{11}$. In breast cancer $\mathrm{T} 47$ cell line decreases in miR-21, miR-15a and miR-141 while increases in miR-200c expression levels when treated with silibinin ${ }^{12}$. Silybin shows anticancer effect on both androgen-dependent and androgen-independent prostate cancer by inhibiting cell growth, cell invasion and metastasis. The epithelial tomesenchymal transitions are targeted by silybin in which epithelial characteristics are stimulated and the expressions of mesenchymal markers are inhibited. Silybin treatment for prostate cancer resulted in cytokeratin-18 up regulation and viment down regulation ${ }^{13}$. Silybin shows time and dose dependent apoptotic action on human bladder transitional cell carcinoma (TCC) which is related to cleavage of caspase 3 and poly(ADP-ribose) polymerase ${ }^{14}$.

The most active component of silymarin i.e silybin contains anti-inflammatory properties by inhibiting the prostaglandins and leukotrienes from polyunsaturated fatty acids in the liver and enzyme lipoxygenase. The potential of silybin is suggested in the treatment of Alzheimer by inhibiting Hsp 90 which leads to degradation of Hsp 90 protein client. Silybin shows an antifibrotic effect by reducing the transformation of stellate cells into myofibroblasts and down regulates gene expression of extracellular matrix components indispensable for fibrosis. Silybin treatment resulted to decrease in CDK2 and CDK4 levels, the apoptosis of ECV304 cells are induced and angiogenesis inhibited by modulation of caspases, Bcl-2 family and NF-kappaB. The growths of some cancers in rodents are inhibited by dietary silybin and its potential is suggested in treatment of cancer. 


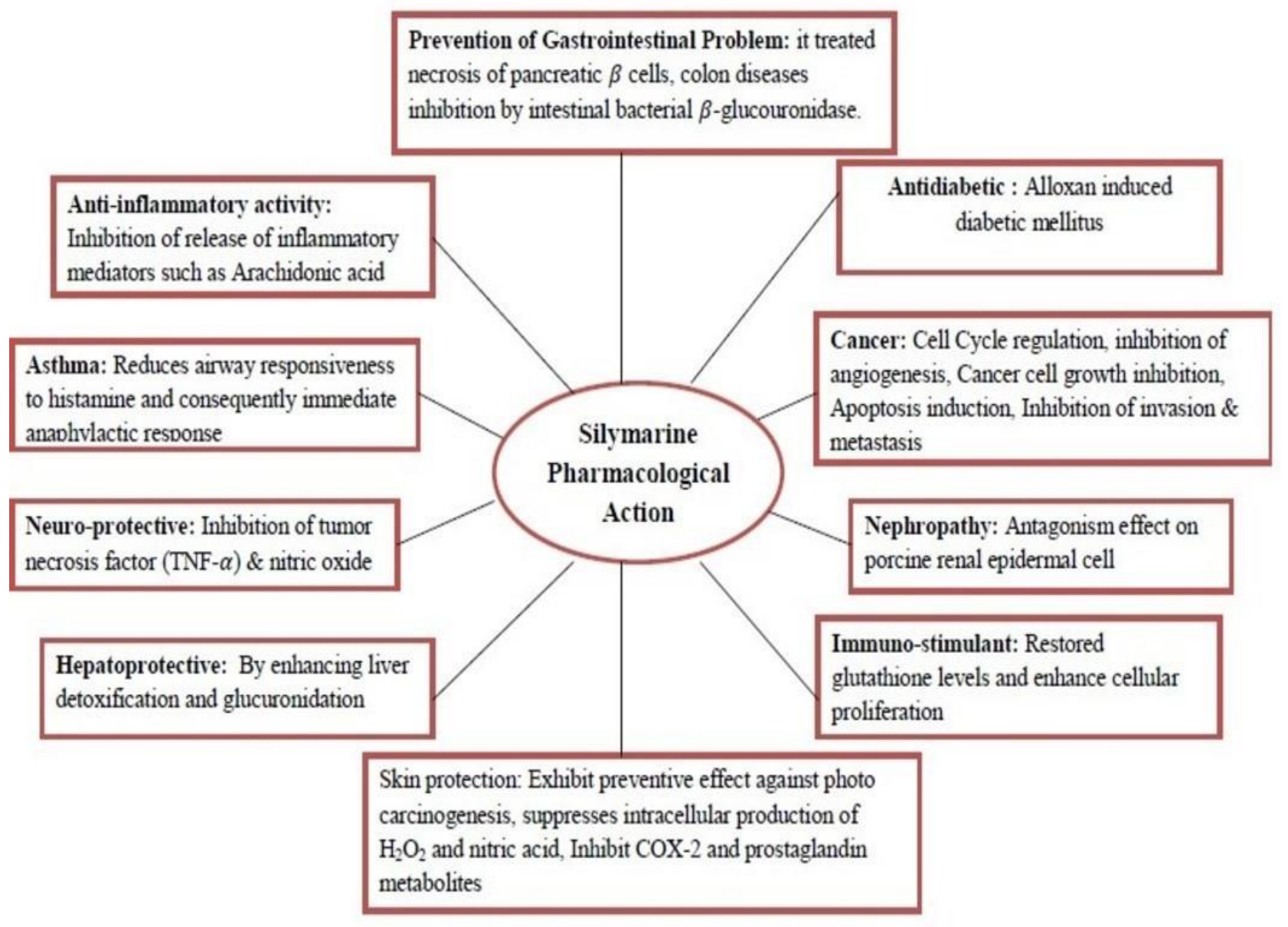

Figure 2: Pharmacological action of silymarin

\section{APPLICATIONS BY DIFFERENT ROUTE OF ADMINISTRATION}

\section{Oral route}

Oral route of drug administration has been known for decades and widely accepted method for drug delivery because of its simple, convenient, noninvasive, safest, and most economical aspect. The challenging problems in oral drug delivery includes, difficulty in swallowing pill, irritant and unpalatable drugs being not suitable for administration by this route, poor stability in the gastric environment, low solubility and poor bioavailability, slow onset of action, less or no control over drug release, nonspecific delivery site and systemic side effects. The Silymarin is BCS class II drug having poor bioavailability due to extensive phase II metabolism, poor aqueous solubility, low permeability across intestinal epithelial cells and rapid excretion in bile and urine. To overcome this problems the different types of silymarin nanoparticles (NPs), such as liposomes, nano- or micro- emulsions, polymeric NPs and solid lipid NPs, polymer conjugates, nanocrystals, polymeric micelles, mixed micelles inclusion complex, continue to be developed in order to improve the stability and bioavailability of Silymarin.

Table 1: Oral drug delivery of silymarin

\begin{tabular}{|c|c|c|c|c|}
\hline Formulation & Composition & Preparation method & Purpose & Ref \\
\hline & & TABLETS & & \\
\hline Floating Tablet & $\begin{array}{l}\text { Hydroxypropyl methyl cellulose, } \\
\text { microcrystalline cellulose, } \\
\text { crospovidone }\end{array}$ & Wet granulation & Prolong gastric residence time & 15 \\
\hline $\begin{array}{l}\text { Erodible matrix } \\
\text { tablets }\end{array}$ & $\begin{array}{l}\text { Glyceryl monostearate, Polyethylene } \\
\text { glycol 6000, Poloxamer188 }\end{array}$ & Melt Fusion & Controlled release of SLM & 16 \\
\hline $\begin{array}{l}\text { Solid dispersion } \\
\text { tablet }\end{array}$ & $\begin{array}{l}\text { Hydroxy propyl- } \beta \text {-cylcodextrine } \\
\text { (HP- } \beta \text {-CD) }\end{array}$ & Direct compression & $\begin{array}{l}\text { Enhance dissolution and oral } \\
\text { bioavailability }\end{array}$ & 17 \\
\hline Osmotic tablets & Cellulose acetate & Melt fusion & Controlled release of SLM & 18 \\
\hline Fast dissolving & Cross povidone, & Dry granulation & $\begin{array}{l}\text { Fast dissolving with improve } \\
\text { patient compliance and }\end{array}$ & 19 \\
\hline
\end{tabular}




\begin{tabular}{|c|c|c|c|c|}
\hline Formulation & Composition & Preparation method & Purpose & Ref \\
\hline tablet & $\begin{array}{l}\text { Microcrystalline Cellulose, } \\
\text { Croscarmellose } \\
\text { sodium, Aerosil }\end{array}$ & & convenience & \\
\hline $\begin{array}{l}\text { Microporous } \\
\text { osmotic pump } \\
\text { tablet }\end{array}$ & $\begin{array}{l}\text { Dibutyl phthalate, soyabean lecithin, } \\
\text { Sodium chloride, lactose, mannitol }\end{array}$ & $\begin{array}{l}\text { Phytosome complex } \\
\text { method }\end{array}$ & $\begin{array}{l}\text { Sustained and controlled- } \\
\text { release drug delivery }\end{array}$ & 20 \\
\hline \multirow[t]{2}{*}{$\begin{array}{l}\text { Nanosuspension } \\
\text { tablet }\end{array}$} & $\begin{array}{l}\text { Polyvinyl alcohol, Tween } 80 \text {, } \\
\text { mannitol }\end{array}$ & Lyophilization & Immediate release & 21 \\
\hline & & LIPOSOMES & & \\
\hline Liposome & Cholesterol & Ethanol injection & $\begin{array}{l}\text { Enhance hepatoprotective and } \\
\text { gastroprotective effect }\end{array}$ & 22 \\
\hline Liposome & $\begin{array}{l}\text { Lecithin, cholesterol, stearyl amine, } \\
\text { tween-80 }\end{array}$ & $\begin{array}{l}\text { Reverse evaporation } \\
\text { technique }\end{array}$ & $\begin{array}{l}\text { Enhance hepatoprotective } \\
\text { effect }\end{array}$ & 23 \\
\hline Liposome & $\begin{array}{l}\rho \text { - amino phenyl- } \beta \text {-D- } \\
\text { Galactopyranoside }\end{array}$ & $\begin{array}{l}\text { Reverse evaporation } \\
\text { technique }\end{array}$ & Targeting to hepatocyte & 24 \\
\hline Liposome & Lecithin, Cholesterol & $\begin{array}{l}\text { Lipid film hydration } \\
\text { method }\end{array}$ & $\begin{array}{l}\text { Targeting to hepatocyte and to } \\
\text { improve oral bioavailability }\end{array}$ & 25 \\
\hline Liposome & $\begin{array}{l}\text { Soybean phosphatidylcholine, } \\
\text { sodium glycocholate }\end{array}$ & $\begin{array}{l}\text { supercritical fluid } \\
\text { technology }\end{array}$ & $\begin{array}{l}\text { improve the dissolution and } \\
\text { bioavailability of silymarin }\end{array}$ & 26 \\
\hline Proliposomes & $\begin{array}{l}\text { Soy-lecithin, } \\
\text { D-Galactosamine, Superoxide } \\
\text { dismutase (SOD), } \\
\text { malondialdehyde (MDA), } \\
\text { glutathione peroxidase (GSH-PX) }\end{array}$ & $\begin{array}{l}\text { simple dissolving } \\
\text { process }\end{array}$ & $\begin{array}{l}\text { improve bioavailability and } \\
\text { hepaptoprotective effects }\end{array}$ & 27 \\
\hline Proliposomes & Phospholipid and mannitol & Film deposition & Enhanced bioavilability & 28 \\
\hline Proliposomes & $\begin{array}{l}\text { soybean phospholipids, cholesterol, } \\
\text { isopropyl myristate and sodium } \\
\text { cholate }\end{array}$ & $\begin{array}{l}\text { Film dispersion-freeze } \\
\text { drying method }\end{array}$ & Improve oral bioavailability & 29 \\
\hline \multirow[t]{2}{*}{ Bilosomes } & $\begin{array}{l}\text { Soybean lecithin } \\
\text { phosphatidylcholine, cholesterol, } \\
\text { Sodium deoxycholate, } \\
\text { Sodium taurocholate, Carbon } \\
\text { tetrachloride }\end{array}$ & $\begin{array}{l}\text { Film hydration } \\
\text { technique }\end{array}$ & $\begin{array}{l}\text { Increase the hepatoprotective } \\
\text { activity of the drug }\end{array}$ & 30 \\
\hline & & $\begin{array}{l}\text { SOLID- LIPID } \\
\text { NANOPARTICLE }\end{array}$ & & \\
\hline $\begin{array}{l}\text { Solid lipid } \\
\text { nanoparticle }\end{array}$ & - & $\begin{array}{l}\text { High pressure } \\
\text { homogenization }\end{array}$ & Enhanced biodistribution & 31 \\
\hline $\begin{array}{l}\text { Solid lipid } \\
\text { nanoparticle }\end{array}$ & ATO 5, lecithin, tween 80 & $\begin{array}{l}\text { High pressure } \\
\text { homogenization }\end{array}$ & Improve oral bioavailability & 32 \\
\hline $\begin{array}{l}\text { Solid lipid } \\
\text { nanoparticle }\end{array}$ & $\begin{array}{l}\text { Compritol } 888 \text { AT0, soyabean } \\
\text { lecithin, Poloxamer-188 }\end{array}$ & $\begin{array}{l}\text { Hot and cold } \\
\text { homogenization }\end{array}$ & $\begin{array}{l}\text { Enhanced biodistribution and } \\
\text { bioavailability }\end{array}$ & 33 \\
\hline \multirow[t]{2}{*}{$\begin{array}{l}\text { Solid lipid } \\
\text { nanoparticle }\end{array}$} & Campritol 888 AT0 & Homogenization & Enhance oral bioavailability & 34 \\
\hline & & SOLID DISPERSION & & \\
\hline Solid Dispersion & Polyethylene glycol 6000 & Fusion method & $\begin{array}{l}\text { Enhanced dissolution rate and } \\
\text { oral bioavailability }\end{array}$ & 35,36 \\
\hline Solid Dispersion & $\begin{array}{l}\text { Hydroxypropyl methyl cellulose E } \\
15 \mathrm{LV},\end{array}$ & $\begin{array}{l}\text { spray drying and co- } \\
\text { precipitation methods }\end{array}$ & Enhance silymarin dissolution & 37 \\
\hline Solid Dispersion & Polyvinylpyrrolidone & Fluid bed technique & Enhance dissolution rate & 38 \\
\hline Solid dispersion & Polyvinylpyrrolidone & Fluid bed techniques & Enhance oral bioavailability & 39 \\
\hline Solid Dispersion & Polyvinylpyrrolidone K30, & supercritical fluids & improve the dissolution and & 40 \\
\hline
\end{tabular}




\begin{tabular}{|c|c|c|c|c|}
\hline Formulation & Composition & Preparation method & Purpose & Ref \\
\hline & $\begin{array}{l}\text { Hydroxypropyl methyl cellulose K4M } \\
\text { and hydroxypropyl methyl cellulose } \\
\text { K15M, Carbon dioxide }\end{array}$ & method & bioavailability & \\
\hline Solid Dispersion & $\begin{array}{l}\text { Hydroxypropyl methylcellulose } \\
\text { (HPMC) }\end{array}$ & $\begin{array}{l}\text { Kneading, spray drying, } \\
\text { coprecipitation }\end{array}$ & Enhance dissolution rate & 41 \\
\hline \multirow[t]{2}{*}{ Solid dispersion } & Tween 80, polyvinylpyrolidone & Spray drying method & $\begin{array}{l}\text { Enhance oral bioavailability } \\
\text { and dissolution rate }\end{array}$ & 42 \\
\hline & & EMULSION & & \\
\hline Emulsion & $\begin{array}{l}\text { Poly(lactic-co-glycolic acid) (PLGA), } \\
\text { Polycaprolactone (PCL), Sodium } \\
\text { Alginate, Chitosan, poly(Llactide) } \\
\text { (PLLA), Eudragit, }\end{array}$ & $\begin{array}{l}\text { Membrane } \\
\text { emulsification }\end{array}$ & $\begin{array}{l}\text { Improve encapsulation } \\
\text { efficiency and drug loading. }\end{array}$ & 43 \\
\hline Nanoemulsion & Sefsol-218, tween-80, ethanol & $\begin{array}{l}\text { Spontaneous } \\
\text { emulsification }\end{array}$ & $\begin{array}{l}\text { Enhance bioavailability and } \\
\text { hepatoprotective activity }\end{array}$ & 44 \\
\hline Nanoemulsion & $\begin{array}{l}\text { Sefsol- 218, polyoxyethylene sorbitan } \\
\text { monooleate }\end{array}$ & Titration method & Enhanced oral bioavailability & 45,46 \\
\hline Nanoemulsion & $\begin{array}{l}\text { Sefsol- 218, } \\
\text { Kolliphor RH40, polyethylene glycol } \\
400\end{array}$ & $\begin{array}{l}\text { Aqueous titration } \\
\text { method }\end{array}$ & $\begin{array}{l}\text { efficient carrier for oral } \\
\text { delivery of silymarin against } \\
\text { human hepatocellular } \\
\text { carcinoma without damaging } \\
\text { normal }\end{array}$ & 47 \\
\hline Nanoemulsion & $\begin{array}{l}\text { Labrasol ECH, Capryol 90, Transcutol } \\
\text { HP, Labrafil, oleic acid, Cremophor } \\
\text { EL, Triacetin }\end{array}$ & $\begin{array}{l}\text { Aqueous titration } \\
\text { method }\end{array}$ & $\begin{array}{l}\text { Improve solubility and oral } \\
\text { absorption of silymarin }\end{array}$ & 48 \\
\hline Nanoemulsion & $\begin{array}{l}\text { Capryol 90, Solutol HS 15, Transcutol } \\
\text { HP }\end{array}$ & $\begin{array}{l}\text { High pressure } \\
\text { homogenization }\end{array}$ & Enhance oral bioavailability & 49 \\
\hline SNEDDS & $\begin{array}{l}\text { Campul GMO, Tween 20, } \\
\text { Crehmophore RH40, Transcutol HP }\end{array}$ & Phase titration method & $\begin{array}{l}\text { improve the dissolution, } \\
\text { permeability, and oral } \\
\text { bioavailability }\end{array}$ & 50 \\
\hline \multirow[t]{2}{*}{ SNEDDS } & $\begin{array}{l}\text { PEG 200, PEG 400, glyceryl } \\
\text { monooleate, polysorbate } 20 \text { and } \\
\text { polyoxyethy- lene-50-hydrogenated } \\
\text { castor oil (HCO-50), Transcutol }\end{array}$ & Phase titration method & $\begin{array}{l}\text { Enhance bioavailability and } \\
\text { dissolution rate }\end{array}$ & 51 \\
\hline & & MICROPARTICLES & & \\
\hline Microparticles & $\begin{array}{l}\text { Lecithin, tween-20, tween-80, span- } \\
\text { 20, propylene glycol }\end{array}$ & $\begin{array}{l}\text { Low energy } \\
\text { emulsification } \\
\text { techniques }\end{array}$ & $\begin{array}{l}\text { Enhanced dissolution, } \\
\text { therapeutic efficiency and } \\
\text { bioavailability }\end{array}$ & 52,53 \\
\hline Microparticles & $\begin{array}{l}\text { Phospholipid, ethanol, sodium } \\
\text { cholate, disodium hydrogen } \\
\text { phosphate, polyvinylpyrrolidone K30 }\end{array}$ & Freez drying & Improve oral bioavailability & 54 \\
\hline \multirow[t]{2}{*}{$\begin{array}{l}\text { Floating } \\
\text { microsphere }\end{array}$} & $\begin{array}{l}\text { Hydroxypropyl methyl cellulose, } \\
\text { microcrystalline cellulose, } \\
\text { crosspovidone }\end{array}$ & Wet granulation & Prolong gastric residence time & 55 \\
\hline & & $\begin{array}{l}\text { NANO STRUCTURED } \\
\text { LIPID CARRIER }\end{array}$ & & \\
\hline $\begin{array}{l}\text { Nanostructured } \\
\text { lipid carrier }\end{array}$ & $\begin{array}{l}\text { Glycerol, monostearate, oleic acid, } \\
\text { tween } 80, \text { caprylic acid, cetyl } \\
\text { palmitate, stearic acid }\end{array}$ & $\begin{array}{l}\text { Emulsification and } \\
\text { ultrasonication method }\end{array}$ & $\begin{array}{l}\text { Improve solubility, stability } \\
\text { and oral bioavailability }\end{array}$ & 56 \\
\hline $\begin{array}{l}\text { Nanostructure } \\
\text { lipid carrier }\end{array}$ & Stearic acid, capryol 90, Brij S20, & $\begin{array}{l}\text { Emulsion evaporation } \\
\text { method }\end{array}$ & $\begin{array}{l}\text { Improve solubility and } \\
\text { absorption of silymarin }\end{array}$ & 57 \\
\hline $\begin{array}{l}\text { Binary lipids- } \\
\text { based } \\
\text { nanostructured } \\
\text { lipid Carriers }\end{array}$ & $\begin{array}{l}\text { Oleic acid, Tween-80, Precirol ATO 5, } \\
\text { egg phosphatidylcholine }\end{array}$ & $\begin{array}{l}\text { Hot high- pressure } \\
\text { homogenization }\end{array}$ & $\begin{array}{l}\text { improve the oral } \\
\text { bioavailability of silymarin }\end{array}$ & 58 \\
\hline Nanostructured & Capryol 90, Lauroglycol 90, oleic acid, & Emulsion evaporation & $\begin{array}{l}\text { Improve solubility and } \\
\text { enhance intestinal }\end{array}$ & 59 \\
\hline
\end{tabular}




\begin{tabular}{|c|c|c|c|c|}
\hline Formulation & Composition & Preparation method & Purpose & Ref \\
\hline \multirow[t]{2}{*}{ lipid carrier } & precirol ATO 5, cetyl palmitate & method & permeability & \\
\hline & & NANOCRYSTALS & & \\
\hline Nanocrystals & Hydroxypropyl- $\beta$-CyD & $\begin{array}{l}\text { high pressure } \\
\text { crystallization }\end{array}$ & $\begin{array}{l}\text { enhanced dissolution rate and } \\
\text { absorbability }\end{array}$ & 60 \\
\hline \multirow[t]{2}{*}{ Nanocrystals } & $\begin{array}{l}\text { acetone, acetonitrile, ethanol and } \\
\text { methanol }\end{array}$ & Precipitation method & $\begin{array}{l}\text { enhance oral bioavailability, } \\
\text { and improve solubility }\end{array}$ & 61 \\
\hline & & NANOPARTICLES & & \\
\hline $\begin{array}{l}\text { Eudragit loaded } \\
\text { nanoparticles }\end{array}$ & $\begin{array}{l}\text { Eudragit RL100, Polyvinyl alcohol, } \\
\text { Hydroxypropyl methyl cellulose }\end{array}$ & $\begin{array}{l}\text { nanoprecipitation } \\
\text { technique }\end{array}$ & $\begin{array}{l}\text { Improve the poor } \\
\text { bioavailability of silymarin } \\
\text { through buccal delivery. }\end{array}$ & 62 \\
\hline $\begin{array}{l}\text { porous silica } \\
\text { nanoparticles }\end{array}$ & $\begin{array}{l}\text { Octylphenol polyoxyethylene, } \\
\text { cyclohexane, a-naphthol }\end{array}$ & $\begin{array}{l}\text { microemulsion and } \\
\text { ultrasonic corrosion } \\
\text { methods }\end{array}$ & $\begin{array}{l}\text { improve oral } \\
\text { bioavailability }\end{array}$ & 63 \\
\hline Nanoparticles & $\begin{array}{l}\text { Hydroxypropyl methyl cellulose, } \\
\text { hydroxy propyl- } \beta \text {-cyclodextrin (HP- } \\
\beta \text {-CD), ethanol }\end{array}$ & Freez drying method & $\begin{array}{l}\text { Improve solubility and } \\
\text { bioavailability }\end{array}$ & 64 \\
\hline Nanoparticles & $\begin{array}{l}\text { Transcutol HP, polysorbate } 80 \\
\text { (Tween 80), castor oil, and } \\
\text { polyvinylpyrrolidone (PVP K30) }\end{array}$ & Spray drying techniques & $\begin{array}{l}\text { enhanced oral bioavailability } \\
\text { and provide excellent hepatic } \\
\text { protection }\end{array}$ & 65 \\
\hline \multirow[t]{2}{*}{ Nanoparticles } & Poloxamer 188, mannitol & $\begin{array}{l}\text { Emulsion solvent } \\
\text { evaporation and freez } \\
\text { drying method }\end{array}$ & $\begin{array}{l}\text { Improve poor aqueous } \\
\text { solubility }\end{array}$ & 66 \\
\hline & & INCLUSION COMPLEX & & \\
\hline $\begin{array}{l}\text { Inclusion } \\
\text { complex }\end{array}$ & $\beta$ - cyclodextrine & Kneading method & $\begin{array}{l}\text { Enhanced dissolution and } \\
\text { bioavailability }\end{array}$ & 67 \\
\hline $\begin{array}{l}\text { Inclusion } \\
\text { complex }\end{array}$ & $\beta$-cyclodextrine & $\begin{array}{l}\text { Kneading, co- } \\
\text { precipitation and } \\
\text { solvent evaporation }\end{array}$ & $\begin{array}{l}\text { Enhanced dissolution and } \\
\text { solubility }\end{array}$ & 68 \\
\hline \multirow[t]{2}{*}{$\begin{array}{l}\text { Inclusion } \\
\text { complex }\end{array}$} & Fulvic acid & $\begin{array}{l}\text { Physical mixing and } \\
\text { kneading methods }\end{array}$ & $\begin{array}{l}\text { improve the solubility and } \\
\text { dissolution profile }\end{array}$ & 69 \\
\hline & & NANOMICELLES & & \\
\hline Nanomicelles & Soluplus & Thin film method & $\begin{array}{l}\text { Improve the solubility and oral } \\
\text { absorption }\end{array}$ & 59 \\
\hline Nanomicelles & $\begin{array}{l}\text { Soluplus, } \\
\text { d- } \alpha \text {-tocopherol, polyethylene glycol } \\
1000 \text { succinate }\end{array}$ & Thin film method & $\begin{array}{l}\text { Improve the solubility and oral } \\
\text { absorption }\end{array}$ & 59 \\
\hline
\end{tabular}

\section{Topical route:}

The topical route has attracted attention due to its ability to deliver drug substance more selectively to a specific site, avoidance of gastric irritation, avoiding drug levels fluctuations, prevents metabolism of drug, improved compliance, and an enhanced suitability for self-medication. The topical route of administration provides the delivery of drug for both local and systemic effects. The major obstacle for topical delivery is stratum corneum and barrier to the penetration of many drug substances. The topical application of silymarin has received attention because of its pharmacological properties such as antioxidant, antiinflammatory, and immunomodulatory properties which may prevent UV-induced skin disorders like skin cancer, erythema, and photoaging. The one of the most challenging aspect of drug development is poor aqueous solubility of silymarin $(3.2 \mathrm{mg} / 100 \mathrm{ml})$. To overcome this issues nanogel, gels, creams, lotions, microemulsion, dendrimer are developed in order to improve solubility, stability and to enhance penetration ability of silymarin. 
Table 2: Topical drug delivery of silymarin

\begin{tabular}{|c|c|c|c|c|}
\hline Formulation & Composition & Preparation Method & Purpose & Ref \\
\hline & & DENDRIMER & & \\
\hline Dendrimer & $\begin{array}{l}\text { Glycine, proline, lysine, } \\
\text { Dimethylformamide, } \\
\text { dichloromethane, trifluoroacetic acid }\end{array}$ & Co-precipitation & $\begin{array}{l}\text { Enhance skin penetration } \\
\text { and deposition }\end{array}$ & 70 \\
\hline \multirow[t]{2}{*}{ Dendrimer } & $\begin{array}{l}\text { Polyamidoamine (PAMAM-G4) } \\
\text { polyethylene glycol, folic acid }\end{array}$ & Solvent evaporation & $\begin{array}{l}\text { Deliver the poorly soluble } \\
\text { drug silybin }\end{array}$ & 71 \\
\hline & & GEL & & \\
\hline \multirow[t]{2}{*}{ Organogel } & $\begin{array}{l}\text { Lecithin, Pluronic F127, isopropyl } \\
\text { myristate }\end{array}$ & & Enhance skin penetration & 72 \\
\hline & & LIPOSOME & & \\
\hline \multirow[t]{2}{*}{ Nanoliposomes } & $\begin{array}{l}\text { Egg lecithin, cholesterol, chloroform, } \\
\text { methanol }\end{array}$ & Extrusion method & Enhance penetration & 73 \\
\hline & & $\begin{array}{l}\text { SOLID LIPID } \\
\text { NANOPARTICLES }\end{array}$ & & \\
\hline SLN's & $\begin{array}{l}\text { Glyceryl monostearate, Tween } 80 \text {, } \\
\text { chloroform and methanol }\end{array}$ & Micro- emulsion method & $\begin{array}{l}\text { Improve stability and } \\
\text { enhance permeation }\end{array}$ & 74 \\
\hline \multirow[t]{2}{*}{ SLN's } & $\begin{array}{l}\text { Glyceryl monostearate, tween 80, } \\
\text { solutol HS and loturol F68 }\end{array}$ & Homogenization & Sustain release & 75 \\
\hline & & MICROEMULSION & & \\
\hline \multirow[t]{2}{*}{ Microemulsion } & $\begin{array}{l}\text { Labrasol, Transcutol Glyceryl } \\
\text { monooleate, ethyl oleate, oleic acid, } \\
\text { isopropyl myristate }\end{array}$ & Phase titration method & $\begin{array}{l}\text { Enhance solubility, } \\
\text { stability and penetration }\end{array}$ & 76 \\
\hline & & $\begin{array}{l}\text { NANOSTRUCTURED LIPID } \\
\text { CARRIER }\end{array}$ & & \\
\hline $\begin{array}{l}\text { Nanostructured } \\
\text { lipid carriers }\end{array}$ & Compritol ATO 888, Pluronic F-68 & $\begin{array}{l}\text { Hot high- pressure } \\
\text { homogenization process }\end{array}$ & $\begin{array}{l}\text { Increase } \\
\text { permeation } \\
\text { and reduced toxicity }\end{array}$ & 77 \\
\hline $\begin{array}{l}\text { Topical } \\
\text { nanostructured } \\
\text { lipid carrier }\end{array}$ & $\begin{array}{l}\text { Glycerolmomstearate, oleic acid, } \\
\text { carbopol } 980\end{array}$ & $\begin{array}{l}\text { Hot high pressure } \\
\text { homogenization process }\end{array}$ & $\begin{array}{l}\text { enhanced solubility, } \\
\text { stability and permeation }\end{array}$ & 78 \\
\hline
\end{tabular}

\section{CONCLUSION:}

Silymarin is active phytomedicine obtained from silymarium plant containing therapeutic efficacy against various diseases. The major concern of silymarin is poor bioavailability, low aqueous solubility, high metabolism, rapid excretion. The other obstacle is route of administration (oral and topical route) which hindered potential of silymarin. The challenging problems in route of administration are low solubility, poor stability in the gastric environment, poor bioavalability, less penetration. To overcome these issues the review focuses on several novel drug delivery strategies such as liposomes, solid lipid nanoparticles, nano or micro-emulsions, microspheres, nanogels, solid dispersions, nanocrystals, nano structured lipid carrier, inclusion complex, dendrimer, micelles have been described to enhance bioavailability, increase solubility and delivery of silymarin. There are still many challenges that need to be resolved such as safety of nanoparticles for long-time, interaction with biological systems and patientfriendly delivery system.

\section{REFERENCES:}

1. Shakeel F, Anwer K, Shazly GA, Jamil S, Measurement and correlation of solubility of bioactive compound silymarin in five different green solvents at $298.15 \mathrm{~K}$ to $333.15 \mathrm{~K}$, Journal of Molecular Liquids, 2014; 195:255-258.

2. Karkanis A, Bilalis D, Efthimiadou A, Cultivation of milk thistle ( Silybum marianum L . Gaertn.), a medicinal weed, Industrial Crops Products, 2011; 34(1):825-830.

3. Kim S, Choi MG, Lee HS, Lee SK, Kim SH, Kim WW, Silibinin Suppresses TNF-a-Induced MMP-9 Expression in Gastric Cancer Cells through Inhibition of the MAPK Pathway, Molecules, 2009; 14:4300-4311.

4. Morazzoni P, Montalbetti A, Malandrino S, Pifferi G, Comparative pharmacokinetics of silipide and silymarin in rats, European Journal of Drug Metabolism and Pharmacokinetics, 1993; 18(3):289-297.

5. Wu J, Lin L, Tsai T, Drug - drug interactions of silymarin on the perspective of pharmacokinetics, Journal of Ethnopharmacology, 2009; 121:185-193.

6. Janc P, Anzenbacherova E, Papous B, Lemr K, Luz P, Veinlichova A, Silybin Is Metabolized by Cytochrome P450 2C8 in Vitro, Drug Metabolism and Disposition, 2007; 35(11):2035-2039.

7. Wu J, Lin L, Hung S, Chi C, Tsai T. Analysis of silibinin in rat plasma and bile for hepatobiliary excretion and oral 
bioavailability application, Journal of Pharmaceutical and Biomedical Analysis, 2007; 45:635-641.

8. Tyler V, Phytomedicines in Western Europe: Their potential impact on herbal medicine in the United States, Herbalgram;1994;30:24-30

9. Decarli LM, Silymarin Retards the Progression of AlcoholInduced Hepatic Fibrosis in Baboons, Journal of Clinical Gastroenterology, 2003; 37:336-339.

10. Zaulet M, Elvira S, Kevorkian M, Dinescu S, Cotoraci C, Suciu M, Protective effects of silymarin against bisphenol A-induced hepatotoxicity in mouse liver, Experimental and Therapeutic Medicine, 2017; 13:821-828.

11. Jiang $\mathrm{K}$, Wang $\mathrm{W}$, Jin $\mathrm{X}$, Wang Z, Ji Z, Meng G, Silibinin , a natural flavonoid, induces autophagy via ROS-dependent mitochondrial dysfunction and loss of ATP involving BNIP3 in human MCF7 breast cancer cells, Oncology, 2015; 33:27112718.

12. Elmira S, Rouholamini $\mathrm{Y}$, Moghassemi S, Maharat Z, Hakamivala A, Effect of silibinin-loaded nano-niosomal coated with trimethyl chitosan on miRNAs expression in 2D and 3D models of T47D breast cancer cell line, Artificial cells, Nanomedicine and Biotechnology, 2017; 1401:1-13.

13. Wu K, Zeng JIN, Li LEI, Fan J, Zhang D, Xue YAN, Silibinin reverses epithelial-to-mesenchymal transition in metastatic prostate cancer cells by targeting transcription factors, Oncology Reports, 2010; 23:1545-1552.

14. Tyagi A, Agarwal C, Harrison G, Glode LM, Agarwal R, Silibinin causes cell cycle arrest and apoptosis in human bladder transitional cell carcinoma cells by regulating CDKI-CDKcyclin cascade, and caspase 3 and PARP cleavages, Carcinogenesis, 2004; 25(9):1711-1720.

15. Garg R, Gupta G Das, Preparation and evaluation of gastroretentive floating tablets of Silymarin, Chemical and Pharmaceutical Bulletin, 2009; 57(6):545-549.

16. Lu C, Lu Y, Chen J, Zhang W, Wu W,Synchronized and sustained release of multiple components in silymarin from erodible glyceryl monostearate matrix system, European Journal of Pharmaceutics and Biopharmaceutics, 2007; 66(2):210-219.

17. Nakhat P. D ,Naidu R. A, Babla I. B, Khan S and Yeole P. G, Design and evaluation of silymarin-HP- ? -CD solid dispersion tablets, Indian Journal of Pharmaceutical Sciences, 2007; 69(2):1-12.

18. Xie Y, Lu Y, Qi J, Li X, Zhang X, Han J, Synchronized and controlled release of multiple components in silymarin achieved by the osmotic release strategy, International Journal of Pharmaceutics, 2013; 441(1-2):111-20.

19. B Mohan, S Saini, NS Gill, Formulation and Evaluation of Fast Dissolving Tablet of Silymarin, PharmaTutor, 2014; 2(9):8190.

20. Zeng QP, Liu ZH, Huang AW, Zhang J, Song HT, Preparation and characterization of silymarin synchronized-release microporous osmotic pump tablets, Drug Design Development Therapy, 2016; 10:519-531.

21. Ibrahim AH, Rosqvist E, Smatt J, Ibrahim HM, Ismael HR, Afouna MI, Formulation and optimization of lyophilized nanosuspension tablets to improve the physicochemical properties and provide immediate release of silymarin, International Journal of Pharmaceutics, 2019; 563(2):217227.

22. Maheshwari H, Agarwal R, Patil C, Katare OP, Preparation and pharmacological evaluation of silibinin liposomes, Arzneimittel-Forschung/Drug Research, 2003; 53(6):420427.

23. El-Samaligy MS, Afifi NN, Mahmoud EA, Evaluation of hybrid liposomes-encapsulated silymarin regarding physical stability and in vivo performance, International Journal of Pharmaceutics, 2006; 319(1-2):121-9.

24. Dube D, Khatri K, Goyal AK, Mishra N, Vyas SP, Preparation and evaluation of galactosylated vesicular carrier for hepatic targeting of silibinin, Drug Development and Industrial Pharmacy, 2010; 36(5):547-555.

25. Nitesh Q, Rai A, Reddy ND, Raj PV, Jain P, Deshpande P, Silymarin liposomes improves oral bioavailability of silybin besides targeting hepatocytes, and immune cells, Pharmacological Reports, 2014; 116:1-11.

26. Yang G, Zhao Y, Feng N, Zhang Y, Improved dissolution and bioavailability of silymarin delivered by a solid dispersion prepared using supercritical fluids, Asian Journal of
Pharmaceutical Science, 2015; 1-9.

27. Wang M, Xie T, Chang Z, Wang L, Xie X, Kou Y, A New Type of Liquid Silymarin Proliposome Containing Bile Salts: Its Preparation and Improved Hepatoprotective Effects, Plos One, 2015; (1):1-15.

28. Yan-yu X, Yun-mei S, Zhi-peng C, Qi-neng P, Preparation of silymarin proliposome: A new way to increase oral bioavailability of silymarin in beagle dogs, International Journal of Pharmaceutics, 2006; 319(1-2):162-168.

29. Chu C, Tong S, Xu Y, Wang L, Fu M, Ge Y, Proliposomes for oral delivery of dehydrosilymarin: preparation and evaluation in vitro and in vivo, Nature Publishing Group, 2011; 32:973-980.

30. Mohamed MA, Hasanein AM, A SAA, Improved hepatoprotective activity of Silymarin via encapsulation in the novel vesicular nanosystem bilosomes, Drug Development and Industrial Pharmacy, 2017; 1-32.

31. Guo XH, Luo SN, Wang HY, Zhou L, Xie MH, Ye WZ, Study on the preparation and biodistribution of $99 \mathrm{mTc}$-HMIBP, Nuclear Science Techniques, 2006; 17(5):285-288.

32. Shangguan M, Qi J, Lu Y, Wu W, Comparison of the oral bioavailability of silymarin-loaded lipid nanoparticles with their artificial lipolysate counterparts: implications on the contribution of integral structure, International Journal of Pharmaceutics, 2015; 489:195-202.

33. He J, Hou S, Lu W, Zhu L, Feng J, Preparation, Pharmacokinetics and Body Distribution of Silymarin-Loaded Solid Lipid Nanoparticles After Oral Administration, Journal of Biomedical Nanotechnology, 2007; 3(2):195-202.

34. He J, Hou S, Feng J, Cai B, Effect of particle size on oral absorption of silymarin- loaded solid lipid nanoparticles, China journal of Chinese material medica, 2005; 30(21):16511653.

35. Li FQ, Hu JH, Wang H, Zhu QG, Sun HJ, Cai Z, Relativities between lattice changes and the function of dissolution improvement of poorly soluble drug silymarin based upon PEG 6,000 solid dispersion system, Yao Xue Xue Bao, 2002; 37:294-298

36. Li FQ, Hu JH, Improvement of the dissolution rate of silymarin by means of solid dispersions, Chemical and Pharmaceutical Bulletin, 2004; 52(8):972-973.

37. Dalwadi S, Soni T, Thakkar V, Gandhi T, Silymarin-solid dispersions: Characterization and influence of preparation methods on dissolution, Acta Pharma, 2010; 60(4):427-443.

38. Sun N, Wei X, Wu B, Chen J, Lu Y \& Wu W, Enhanced dissolution of silymarin / polyvinylpyrrolidone solid dispersion pellets prepared by a one-step fluid-bed coating technique, Powder Technology, 2008; 182:72-80.

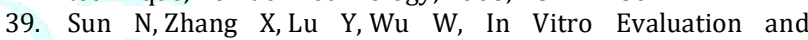
Pharmacokinetics in Dogs of Solid Dispersion Pellets Containing Silybum marianum extract prepared by Fluid-Bed Coating, Planta Medica. 2008; 74:126-132.

40. Yang G, Zhao Y, Feng N, \& Zhang Y, Improved dissolution and bioavailability of silymarin delivered by a solid dispersion prepared using supercritical fluids. Asian Journal of Pharmaceutical Sciences. 2015; 10(3):1-9.

41. Sonali D, Tejal S, Vaishali T, Tejal G. Silymarin-solid dispersions: characterization and influence of preparation methods on dissolution. Acta Pharm. 2010; 60:427-443

42. Shin Y, Bae O, Yousaf AM, Yong CS, Kim JO \& Choi H, A novel solid dispersion system for natural product-loaded medicine: silymarin-loaded solid dispersion with enhanced oral bioavailability and hepatoprotective activity, Journal of Microencapsulation, 2014; 2048:1-8.

43. Piacentini E, Pharmaceutical Particles Design by Membrane Emulsification: Preparation Methods and Applications in Drug Delivery, Current Pharmaceutical Design, 2018; 23(2):302318.

44. Parveen R, Baboota S, Ali J, Ahuja A, Vasudev SS \& Ahmad S, Effects of Silymarin Nanoemulsion against Carbon Tetrachloride- induced Hepatic Damage, Archieves of Pharmacal Research, 2011; 34(5):767-774.

45. Parveen R, Baboota S, Ali J, Ahuja A, Vasudev SS \& Ahmad S, Oil based nanocarrier for improved oral delivery of silymarin: In vitro and in vivo studies, International Journal of Pharmaceutics, 2011; 413(1-2):245-253.

46. Parveen R, Baboota S, Ali J, Ahuja A \& Ahmad S, Stability studies of silymarin nanoemulsion containing Tween 80 as a 
surfactant, Journal of Pharmacy and Bioallied Sciences, 2015; 7(4):321-324.

47. Ahmad U, Akhtar J, Singh SP, Badruddeen B, Ahmad FJ, Siddiqui S \& Wahajuddin W, Silymarin nanoemulsion against human hepatocellular carcinoma: development and optimization, Artificial Cells, Nanomedicine and Biotechnology, 2018; 46(2): 231-241.

48. Piazzini V, Rosseti C, Bigagli E \& Bergonzi MC, Prediction of Permeation and Cellular Transport of Silybum marianum Extract Formulated in a Nanoemulsion by Using PAMPA and Caco-2 Cell Models, Planta Medica, 2017; 83:1184-1193.

49. Nagi A, Iqbal B, Kumar S, Sharma S, Ali J, Baboota S, Quality by design based silymarin nanoemulsion for enhancement of oral bioavailability, Journal of Drug Delivery Science and Technology, 2017; 40:35-44.

50. Shih T \& Yeh T, Self-nanoemulsifying drug delivery systems ameliorate the oral delivery of silymarin in rats with Roux-en$\mathrm{Y}$ gastric bypass surgery, International Journal of Nanomedicine, 2015; 10:2403-2416.

51. Woo JS, Kim T, Park J \& Chi S, Formulation and Biopharmaceutical Evaluation of Silymarin Using SMEDDS, Plos One, 2007;30(1):82-89.

52. Abrol S, Trehan A, Katare OP, Formulation, characterization, and in vitro evaluation of silymarin-loaded lipid microspheres, Drug Delivery, 2004; 11:185-191

53. Abrol S, Trehan A, Katare OP, Comparative study of different silymarin formulations: formulation, characterisation and in vitro/in vivo evaluation, Current Drug Delivery, 2005; 2:45-51

54. Zhu Y, Wang M, Zhang Y, Zeng J, Yu J, \& Xu X, In Vitro Release and Bioavailability of Silybin from Micelle-Templated Porous Calcium Phosphate Microparticles, APPS Pharma Sci Tech, 2015; 17(5): 1-8.

55. Garg R, Gupta GD, Preparation and evaluation of gastroretentive floating tablets of silymarin, Chemical and Pharmaceutical Bulletin, 2009; 57(6):545-549.

56. Chaudhary S, Garg T, Murthy RSR, Rath G and Goyal AK, Development, optimization and evaluation of long chain nanolipid carrier for hepatic delivery of silymarin through lymphatic transport pathway, International Journal of Pharmaceutics, 2015; 14706:1-14

57. Piazzini V, Lemmi B, Ambrosio MD, Cinci L, Luceri C, Bilia AR, \& Bergonzi MC, Nanostructured Lipid Carriers as Promising Delivery Systems for Plant Extracts: The Case of Silymarin, Applied Sciences, 2018; 1163(8):1-15.

58. Shangguan M, Lu Y, Qi J, Han J, Tian Z, Xie Y, Wu W, Binary lipids-based nanostructured lipid carriers for improved oral bioavailability of silymarin, Journal of Biomaterials Applications, 2014; 28(6):887-896.

59. Piazzini V, Ambrosio MD, Luceri C, Cinci L, Landucci E, Bilia $\mathrm{AR}$, \& Bergonzi MC, Formulation of Nanomicelles to Improve the Solubility and the Oral Absorption of Silymarin, Molecules, $2019 ; 24: 1-20$.

60. Onodera R, Hayashi $\mathrm{T}$, Nakamura $\mathrm{T}$, Aibe $\mathrm{K}, \mathrm{T}$ ahara $\mathrm{K}$, \& Takeuchi H, Preparation of silymarin nanocrystals using a novel high-pressure crystallization technique and evaluation of its dissolution and absorption. Asian Journal of Pharmaceutical Sciences, 2016; 11(1):211-212.

61. El-batal AI, Elmenshawi SF, Ali AMA, \& Goodha E. Preparation and Characterization of Silymarin Nanocrystals and Phytosomes with Investigation of their Stability using Gamma Irradiation, Indian Journal of Pharmaceutical Education and Research, 2018; 52(4):174-183.

62. El-Nahas AE, Allam AN, \& El-Kamel A H, Mucoadhesive buccal tablets containing silymarin Eudragit-loaded nanoparticles: formulation, characterisation and ex vivo permeation, Journal of Microencapsulation, 2017; 34(5):463-474.
63. Cao X, Fu M, Wang L, Liu H, Deng W, Qu R, Oral bioavailability of silymarin formulated as a novel 3-day delivery system based on porous silica nanoparticles, Acta Biomater, 2012; 8(6):2104-2112.

64. Wu W, Zu Y, Wang L, Li Y, \& Liu Y, Preparation, characterization and antitumor activity evaluation of silibinin nanoparticles for oral delivery through liquid antisolvent precipitation, RSC Advances, 2017; 7:54379-54390.

65. Bae $\mathrm{O}$ and Kim JO, Silymarin-loaded solid nanoparticles provide excellent hepatic protection: physicochemical characterization and in vivo evaluation, International Journal of Nanomedicine, 2013; 8:3333-3343.

66. Deng Y, Zhang Y, Zu Y, Bolin L, Wu M, Wu W, Silymarin nanoparticles through emulsion solvent evaporation method for oral delivery with high antioxidant activities, bioavailability, and absorption in the liver, RSC Advances, 2016;1-12.

67. Arcari M, Brambilla A, Brandt A, Caponi R, Corsi G, Rella M, Solinas F, Wachter W P, A new inclusion complex of silibinin and beta-cyclodextrins: in vitro dissolution kinetics and in vivo absorption in comparison with traditional formulations, Bollettino Chimico Farmaceutico, 1992; 131(5):205-209.

68. Ghosh A, Biswas S, Ghosh T, Preparation and evaluation of silymarin b-cyclodextrin molecular inclusion complexes, Journal of Young Pharmacists, 2011; 3:205-210.

69. Javed S, Kohil K, \& Ahsan W, Solubility and Dissolution Enhancement of Silymarin with Fulvic Acid, International Journal of Drug Development and Research, 2016; 8(1):9-14.

70. Shetty PK, Manikkath J, Tupally K, Kokil G, Hegde AR, Raut SY, Mutalik S, Skin Delivery of EGCG and Silibinin: Potential of Peptide Dendrimers for Enhanced Skin Permeation and Deposition, AAPS PharmSciTech, 2017; 18(6):2346-2357.

71. Vidal F, Pilar V, Cayum F R, Carola D, Fuentealba J, Aguayo LG ,Guzm L, Prevention of Synaptic Alterations and Neurotoxic Effects of PAMAM Dendrimers by Surface Functionalization, Nanomaterials, 2007; 8(1):1-12.

72. Mady FM, Essa H, El-Ammawi T, Abdelkader H \& Hussein AK. Formulation and clinical evaluation of silymarin pluroniclecithin organogels for treatment of atopic dermatitis, Drug Design, Development and Therapy, 2016;10:1101-1110

73. Zohreh F, Amir G, Masoud G, In-vitro and In-vivo Evaluation of Silymarin Nanoliposomes against Isolated Methicillinresistant Staphylococcus aureus, Iranian Journal of Pharmaceutical Research, 2015; 14(2):627-633.

74. Netto G, Jobin J, Development, characterization, and evaluation of sunscreen cream containing solid lipid nanoparticles of silymarin, Journal of Cosmetic Dermatology, 2017; 17(6):1073-1083.

75. Praphanwittaya P, Chomto P, \& Phaechamud T, Silymarin Solid Lipid Nanoparticle containing Shellac Wax Fabricated with Hot Melt Emulsification, Journal of Pharmaceutical, Biological and Chemical Sciences, 2013; 4(1):784-801

76. Panapisal V, Charoensri S \& Tantituvanont A, Formulation of microemulsion systems for dermal delivery of silymarin, AAPS PharmSciTech, 2012; 13(2):389-399.

77. Singh P, Arya M, Kanoujia J, Singh M, Gupta KP \& Saraf SA, Design of topical nanostructured lipid carrier of silymarin and its effect on 7,12-dimethylbenz[a] anthracene (DMBA) induced cellular differentiation in mouse skin, RSC Advances, 2016; 6(88):84965-84977.

78. Singh P, Singh M, Kanoujia J \& Arya M, Process optimization and photostability of silymarin nanostructured lipid carriers : effect on UV-irradiated rat skin and SK-MEL 2 cell line, Drug Delivery and Translational Research, 2016; 6:597-609. 